\title{
Editorial
}

Nephrology

Published online: January 24, 2019

DOI: 10.1159/000496275

\section{Albuminuria Regression in Diabetes: A Therapeutic Target for Nephro- and Cardio-Protection, in Clinics and Research}

\author{
Piero Ruggenentia, ${ }^{a}$ Giuseppe Remuzzi ${ }^{a, c}$ \\ a Istituto di Ricerche Farmacologiche Mario Negri IRCCS, Milan, Italy; ${ }^{b}$ Unit of Nephrology and Dialysis, Azienda \\ Socio-Sanitaria Territoriale Papa Giovanni XXIII, Bergamo, Italy; ${ }^{\mathrm{C}}$ L. Sacco Department of Biomedical and Clinical \\ Sciences, University of Milan, Milan, Italy
}

Following the pioneering observation that urinary albumin excretion (UAE) can be increased in "newly detected hyperglycemics" [1], observational studies found that even small increases in UAE below the detection limit of a standard dipstick (microalbuminuria: UAE 20$200 \mu \mathrm{g} / \mathrm{min}$ ) strongly predict future macroalbuminuria (UAE $>200 \mu \mathrm{g} / \mathrm{min}$ ) in type 1 diabetics and cardiovascular death in patients with type 2 diabetes. Albuminuria associates with cardiovascular events even in the range considered to be "normal" (UAE $<200 \mu \mathrm{g} / \mathrm{min}$ ), in diabetic as in nondiabetic individuals. Consistently, in 1,204 type 2 diabetics with normoalbuminuria from the BENEDICT trial, there was a continuous relationship between UAE rate at inclusion and incidence of major cardiovascular events during approximately 10 years of follow-up, with a progressive incremental cardiovascular risk that was already evident at an excretion rate ranging from 1 to $2 \mu \mathrm{g} / \mathrm{min}$ as compared to UAE $<1 \mu \mathrm{g} / \mathrm{min}$ [2]. Thus, as for arterial blood pressure, blood glucose, and serum cholesterol levels, UAE is a continuous risk factor for renal and cardiovascular events. On the other end, UAE $>200 \mu \mathrm{g} /$ min clearly identifies patients with progressive renal disease and a cardiovascular mortality rate that exceeds by

\section{KARGER}

(c) 2019 S. Karger AG, Basel

E-Mail karger@karger.com

www.karger.com/ajn about 6 folds the rate observed in subjects with lower levels of albuminuria or no evidence of urinary abnormalities. Macroalbuminuria invariably associates with clinical (>1 g/24-h) proteinuria and progressive glomerular filtration rate (GFR) decline. Renal insufficiency and further increase of proteinuria to a nephrotic range $(>3.5$ $\mathrm{g} / 24$-h) may cause volume overload and worsen hypertension, dyslipidemia, and endothelial dysfunction, all of which may independently sustain the excess renal and cardiovascular mortality in this context [3].

Post hoc analysis of 23,480 microalbuminuric patients with atherosclerotic vascular disease or diabetes from the TRANSCEND and ONTARGET trials assessing whether albuminuria, in addition to arterial hypertension, hyperglycemia, or dyslipidemia, is a modifiable risk factor suitable for clinical monitoring and targeted intervention found that halving in UAE over a 2-year interval predicted a $15 \%$ reduction in mortality risk during the subsequent 3 years, whereas doubling of UAE was followed by a $30 \%$ increase in cardiovascular events, a $40 \%$ increase in renal events, and a $50 \%$ increase in mortality during the same observation period. These findings confirmed and extended data from the RENAAL trial showing that 
6-month reduction in albuminuria predicted a reduced risk of doubling of serum creatinine, progression to ESKD, cardiovascular events, and death on subsequent follow-up in 1,513 type 2 diabetics with macroalbuminuria. Consistently, the PLANET 1 and II trials showed that proteinuria reduction at 14 weeks predicted slower decline of GFR (indirectly estimated by serum-creatinine-based prediction equations) during the subsequent 9 months of follow-up in patients with diabetic or nondiabetic chronic kidney disease (CKD), respectively.

Evidence that albuminuria reduction is nephro- and cardio-protective can be generalized to the real world of type 2 diabetics, referred to outpatient clinics or general practitioners, would have major implications for clinicians and health-care providers. Approximately $8.5 \%$ of adult subjects worldwide are type 2 diabetics, a prevalence projected to double by 2,030 . Their cardiovascular mortality is twice as high compared to that of nondiabetics and similar to that of patients with a previous myocardial infarction. Almost all type 2 diabetics are hypertensive, and the association between diabetes and hypertension increases the cardiovascular risk by almost 4 times. Concomitant macroalbuminuria and CKD further increase cardiovascular risk and strongly predict progression to ESKD, at least in those patients who do not die from cardiovascular causes before their kidneys fail. One-third to a half of new incident patients requiring chronic renal replacement therapy by dialysis or transplantation in the Western world are affected by type 2 diabetes. Where renal replacement therapy is not available, these patients will die from uremia and related complications. Outcome is even poorer in type 2 diabetics who need chronic insulin therapy to control hyperglycemia. These patients are usually old, have a long-lasting history of diabetes, and a high rate of comorbidities. Moreover, insulin therapy increases the body weight, and overweight or obesity is an additional independent risk factor for renal and cardiovascular complications. Thus, as emphasized by Anyanwagu et al. [4] , insulin-treated type 2 diabetics are a particularly frail population that, however, might also benefit the most from intensified intervention aimed to reduce albuminuria. To formally address this hypothesis, Anyanwagu et al. [4] evaluated the relationships between changes in albuminuria after 1-year follow-up and 5-year all-cause mortality and cardiovascular events in 11,074 insulin-treated type 2 diabetics with macroalbuminuria (defined as albumin to creatinine ratio $>300 \mathrm{mg} / \mathrm{g}$ in spot urine samples) from UK general practices between 2007 and 2014 [4]. In 1,552 patients (14\%) achieving regression of macroalbuminuria to $<300 \mathrm{mg} / \mathrm{g}$, all-cause mortality and stroke events were 31 and $27 \%$ lower, respectively, than in 9,522 patients $(86 \%)$ with persistent or worsening macroalbuminuria, despite similar treatment. These findings lead the authors to conclude that "levels of albuminuria should be considered not only as an important risk marker but also an important therapeutic target for cardiovascular and mortality prevention in patients with type 2 diabetes and should be a key consideration when determining drug choice irrespective of blood pressure and glucose levels."

Unfortunately, despite intensified control of modifiable risk factors, including albuminuria, patients with type 2 diabetes remain at substantial risk of cardiovascular and renal events. Cardiovascular and renal trials with sulodexide, thiazolinediones, dipeptydil peptidase- 4 , and bardoxolone showed no benefit from these novel medications. Moreover, some of them were prematurely closed for safety or futility reasons. Much more encouraging results were observed with glucagone-like peptide- 1 and renal sodium-glucose co-transporter 2 (SGLT2) inhibitors, medications developed to improve metabolic control without favoring weight gain and progressive B-cell failure. In combination with RAS inhibitors, both classes of oral antidiabetic agents reduced a composite endpoint of progression to persistent macroalbuminuria, doubling of serum creatinine, ESKD, or death from renal disease as compared to placebo. But impressive cardiovascular and renal benefits were observed in particular with SGLT2 inhibitors, such as empagliflozin, canagliflozin, and dapagliflozin. The EMPAREG OUTCOME, CANVAS, and DECLARE-TIMI 58 trials found that these medications, in addition to favor weight loss and ameliorate metabolic and blood pressure control, reduced the risk of a series of endpoints, including myocardial infarction, stroke, or cardiovascular and all-cause death, (considered in different combinations and/or as single endpoints) as compared to placebo, in particular, in patients with established cardiovascular disease. Moreover, in a meta-analysis of the 34,322 patients included in these trial [5], SGLT2 inhibitors consistently reduced hospitalization for heart failure or progression of renal disease in the whole study group. A prespecified estimated-GFR slope analysis of the EMPA-REG outcome trial also found that, as previously observed with ACE inhibitors in diabetic and non-diabetic proteinuric $\mathrm{CKD}$, the protective effect against the progressive decline of GFR was largely driven by treatment effect in the subgroup of patients with macroalbuminuria [6]. In patients with overt nephrotic syndrome (urinary albumin to creatinine ratio $\geq 2,200 \mathrm{mg} / \mathrm{g}$ ), between-groups 
difference in the rate of GFR decline averaged $6.1 \mathrm{~mL} /$ $\min / 1.73 \mathrm{~m}^{2}$, and the rate of GFR loss was more than 2 folds slower with empagliflozin than placebo [7]. This effect was preceded by a short-term reduction in GFR and albuminuria. These findings corroborate the hypothesis that, as previously reported for RAS inhibitors, longterm nephroprotection can be explained by amelioration of glomerular hyperfiltration and improved sieving function of the glomerular barrier [3]. Noteworthy, in this particularly frail population, empagliflozin was well tolerated, and its safety profile was similar to that of placebo. In particular, the risk of hyperkalemia, one of the major limitations to ACE inhibitor and ARB combination therapy in particular in patients with diabetes, was similar in the empagliflozin and placebo treatment groups. Thus, combining newer and older agents such as SGLT2 and RAS inhibitors may help to implement more effective intervention strategies for nephro and cardioprotection in millions of people with type 2 diabetes. Controlled trials should assess whether the benefits of combined therapy can be extended to patients with unhealthy obesity or nondiabetic proteinuric CKD and whether SGLT2 inhibitors may be a valuable alternative to RAS inhibitors for those at highest risk of hyperkalemia.

\section{References}

1 Keen H, Chlouverakis C, Fuller J, Jarrett RJ: The concomitants of raised blood sugar: studies in newly-detected hyperglycaemics: II. Urinary albumin excretion, blood pressure and their relation to blood sugar levels. Guy's Hosp Rep 1969;118:247-254.

2 Ruggenenti P, Porrini E, Motterlini N, et al: Measurable urinary albumin predicts cardiovascular risk among normoalbuminuric patients with type 2 diabetes. J Am Soc Nephrol 2012;23:1717-1724.
3 Ruggenenti P, Cravedi P, Remuzzi G: Mechanisms and treatment of CKD. J Am Soc Nephrol 2012;23:1917-1928.

4 Anyanwagu U, Donnelly R, Idris I: Albuminuria regression and all-cause mortality among insulin-treated patients with type 2 diabetes: analysis of a large UK primary care cohort. Am J Nephrol 2019;49:146-155.

5 Zelniker TA, Wiviott SD, Raz I, et al: SGLT2 inhibitors for primary and secondary prevention of cardiovascular and renal outcomes in type 2 diabetes: a systematic review and metaanalysis of cardiovascular outcome trials. Lancet 2018;pii:S0140-6736(18)32590-X.
6 Wanner C, Heerspink HJ, Zinman B, et al: Empagliflozin and kidney function decline in patients with type 2 diabetes: a slope analysis from the EMPA-REG outcome trial. J Am Soc Nephrol 2018;29:2755-2769.

7 Ruggenenti P, Inzucchi SE, Zinman B, et al: Empagliflozin and progression of chronic kidney disease in type 2 diabetes complicated by nephrotic-range proteinuria: insights from the empa-reg outcome ${ }^{\circledR}$ trial. Nephrol Dial Transplant 2018;33(suppl 1):i487. 